\title{
Counting 2-Connected 4-Regular Maps on the Projective Plane
}

\author{
Shude Long* \\ Department of Mathematics \\ Chongqing University of Arts and Sciences, \\ Chongqing 402160, P.R.China \\ longshude@163.com
}

\author{
Han Ren ${ }^{\dagger}$ \\ Department of Mathematics \\ East China Normal University, \\ Shanghai 200062, P.R.China \\ hren@math.ecnu.edu.cn
}

Submitted: Jan 17, 2014; Accepted: Jun 11, 2014; Published: Jun 27, 2014

Mathematics Subject Classifications: 05C10, 05C30, 05C45

\begin{abstract}
In this paper the number of rooted (near-) 4-regular maps on the projective plane are investigated with respect to the root-valency, the number of edges, the number of inner faces, the number of nonroot-vertex-loops, the number of nonroot-vertexblocks. As special cases, formulae for several types of rooted 4-regular maps such as 2-connected 4-regular projective planar maps, rooted 2-connected (connected) 4-regular projective planar maps without loops are also presented. Several known results on the number of 4-regular maps on the projective plane are also concluded. Finally, by use of Darboux's method, very nice asymptotic formulae for the numbers of those types of maps are given.
\end{abstract}

\section{Introduction}

We follow $[7,18,31]$ to define a graph (map). A graph (map) is connected and may have loops or multi-edges (or parallel edges as some people called it). A graph (map) is $k$-connected if it needs at least $k$ vertices to separate the graph (map) [7]. One may see that this definition is slightly different from that given by Tutte [30]. For instance, a 2-connected graph (map) may have loops which have been excluded by Tutte.

A planar map (projective planar map) is a graph $G$ drawn on the sphere $S_{0}$ (the projective plane $N_{1}$ ) such that edges intersect at vertices and each component of $S_{0}-$ $G\left(N_{1}\right)$ is a disc called face. Generally, we may define a map on higher surfaces. A circuit $\mathcal{C}$ on a surface $\Sigma$ is essential (or noncontractible as some people named it) if $\Sigma-\mathcal{C}$ has no component homeomorphic to an open disc. Otherwise it is called planar (or trivial).

\footnotetext{
*Supported by Natural Science Foundation Project of Chongqing grant cstc2012jjA00041 and the Innovation Foundation of Chongqing grant KJTD201321.

${ }^{\dagger}$ Supported by the NNSFC grant 11171114. The corresponding author.
} 
A map $M$ is rooted if an edge $e_{r}(M)$, a vertex $v_{r}$ on the edge and a direction along one side of the edge are all distinguished. All maps here are rooted unless special statements are given. One may see from some pioneer's works $[3,18,31]$ that rooting a map may trivialize the automorphism group and makes it possible to build rooted maps by recursive relations. So the general way for one to count rooted maps of a given type in an exact manner is to set up functional equation(s), usually the order of the equation(s) goes higher with the increase of the genera of the surfaces or some other restrictions (as the connectivity etc.) on the maps (graphs), and then crack the equation(s) in every way. The most successful way of doing so is the so-called quadratic method (or double-root method as some people named it) developed by Brown [8] which enables one to solve a quadratic equation by solving a system of two or more equations. This method almost fails in the maps on higher surfaces. In view of exact enumerating nonplanar maps, some people such as T.W.S. Walsh et al. [35] did some works in a general way. Both D. Arqués [1] and, independently, E.A. Bender et al. [4, 5] counted rooted maps on the torus as a function of the number of edges. Moreover, Gao et al. [13] treated the exact enumeration of rooted 3-connected triangular maps on the projective plane and obtained a simple parametric expression for its generating function of the number of vertices. Since elegant formulae were very difficult to obtain for maps on general surfaces, some people such as E.A. Bender started systematically working on asymptotic formulae. Many scholars such as E.A. Bender et al. [6], G. Chapuy et al. [10], Gao [11, 12], A. Mednykh et al. [22, 23] and T.W.S. Walsh et al. [36] have investigated many types of maps on general surfaces and gotten asymptotic evaluations of nonplanar maps up to now. For a survey one may see [3] or [17].

A (rooted) near-4-regular map is such one having all the vertices 4-valent except possibly the rooted one. It is clear that a near-4-regular map is Eulerian. A map is called near-simple if no loops or multi-edges are permitted except possibly only two parallel edges containing the root-vertex.

4-regular maps are very important for applications in many fields such as rectilinear embedding in VLSI, the Gaussian crossing problem in graph theory, the knot problem in topology and the enumerations of some other types of maps [18-21]. Rooted (near-) 4regular maps (or their dual: quadrangulations) have been investigated by many scholars. We list them (as far as we know) as follows:

(1) rooted bicubic maps [32];

(2) rooted trees [33];

(3) rooted quadrangulations [9];

(4) rooted c-nets via quadrangulations [24];

(5) rooted one-faced maps on surfaces [35, pp.212-213];

(6) rooted $2 d$-regular maps on all surfaces [15];

(7) rooted 4-regular planar maps [18, pp.159-166];

(8) rooted near-4-regular planar Eulerian trials [27];

(9) rooted loopless 4-regular maps on the projective plane, torus and the Klein bottle [25-28];

(10) rooted 2-connected 4-regular maps on the plane [29]. 
We expect that several more classes of 4-regular maps could be added into this list. This is main aim of this paper.

Remark. Here we regard planar trees or more generally: maps with one face on surfaces, some people also called them monopoles, as a special kind of near-4-regular maps.

\section{A general equation for maps on $N_{1}$}

In this section we shall set up a general equation with up to six more parameters for rooted near-4-regular maps on the projective plane which will imply several new results for some classes of maps unhandled before and conclude several known results cited in the list above. But first we should give some more definitions on maps.

Let $\mathcal{U}$ and $\mathcal{U}_{p}$, respectively, denote the set of all rooted near-4-regular maps on the plane and the projective plane. Let their enumerating functions be, respectively,

$$
\begin{aligned}
f(x, y, z, t, w, q) & =\sum_{M \in \mathcal{U}} x^{2 m(M)} y^{s(M)} z^{n(M)} t^{\alpha(M)} w^{\beta(M)} \\
f_{p}(x, y, z, t, w, q) & =\sum_{M \in \mathcal{U}_{p}} x^{2 m(M)} y^{s(M)} z^{n(M)} t^{\alpha(M)} w^{\beta(M)}
\end{aligned}
$$

where the variables $x, y, z, t, w$ and $q$ mark, respectively, the root-valency, the number of edges, the number of inner faces, the number of nonroot-vertex-loops, the number of cut-vertices other than the rooted one.

The set $\mathcal{U}_{p}$ may be partitioned into three parts as

$$
\mathcal{U}_{p}=\mathcal{U}_{p 1}+\mathcal{U}_{p 2}+\mathcal{U}_{p 3}
$$

where

$$
\begin{aligned}
& \mathcal{U}_{p 1}=\left\{M \mid e_{r}(M) \text { is a planar loop }\right\}, \\
& \mathcal{U}_{p 2}=\left\{M \mid e_{r}(M) \text { is an essential loop }\right\}, \\
& \mathcal{U}_{p 3}=\left\{M \mid e_{r}(M) \text { is a link }\right\} .
\end{aligned}
$$

Lemma 1. Let $\mathcal{U}_{<p 1>}=\left\{M-e_{r}(M) \mid M \in \mathcal{U}_{p 1}\right\}$. Then

$$
\mathcal{U}_{<p 1>}=\mathcal{U} \odot \mathcal{U}_{p}+\mathcal{U}_{p} \odot \mathcal{U}
$$

where " $\odot$ " is the $1 v$-production of the sets of maps defined in [18, pp.88-89].

Proof. For a map $M \in \mathcal{U}_{p}$, the root-edge $e_{r}(M)$ is a planar loop. The inner and outer regions determined by $e_{r}(M)$ are, respectively, two elements of $\mathcal{U}$ and $\mathcal{U}_{p}$. Since this procedure is reversible, the lemma follows.

By the above lemma, the enumerating function of $\mathcal{U}_{1}$ is

$$
f_{p 1}=2 x^{2} y z f f_{p}
$$


Since maps in $\mathcal{U}_{p 2}$ are in fact obtained from planar ones by making a twist at the rootvertex and then introducing a loop with only one side and the operation is reversible, after a similar procedure as [25] we have the enumerating function of $\mathcal{U}_{p 2}$ as

$$
f_{p 2}=x^{2} y \frac{\partial(x f)}{\partial x} .
$$

The following result is easy to obtain from the definition.

Lemma 2. Let $\mathcal{U}_{(p 3)}=\left\{M \bullet e_{r}(M) \mid M \in \mathcal{U}_{p 3}\right\}$. Then $\mathcal{U}_{(p 3)}=\mathcal{U}_{p}-\mathcal{U}_{p}(2)$, where $\mathcal{U}_{p}(2)$ is the set of maps in $\mathcal{U}_{p}$ whose root-valencies are all 2 , where $M \bullet e_{r}(M)$ denotes the rooted map after contracting the root-edge $e_{r}(M)$ of a map $M$.

By Lemma 2, the enumerating function of $\mathcal{U}_{(p 3)}$ is $f_{(p 3)}=f_{p}-x^{2} F_{p}(2)$, where $F_{p}(2)$ is the enumerating function of $\mathcal{U}_{p}(2)$.

Since splitting the root-vertex may create nonroot-vertex loops, the set $\mathcal{U}_{(p 3)}$ has to be divided into several more parts as

$$
\mathcal{U}_{(p 3)}=\sum_{i=1}^{6} \mathcal{U}_{(p 3)}^{i},
$$

where maps in $\mathcal{U}_{(p 3)}^{i}(1 \leqslant i \leqslant 5)$ have the structures as depicted in Figure 1 , where the shadowed regions represent a map on $N_{1}$ or $S_{0}$.

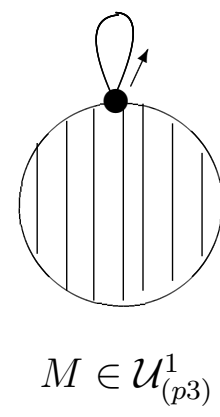

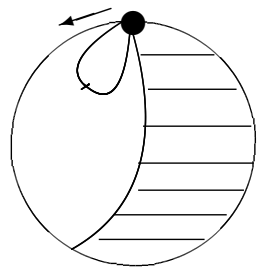

$M \in \mathcal{U}_{(p 3)}^{2}$

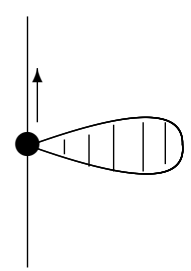

$M \in \mathcal{U}_{(p 3)}^{3}$

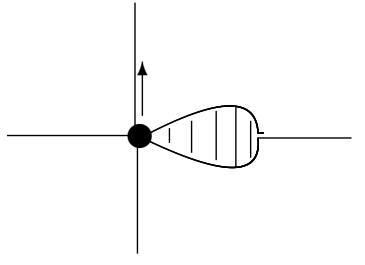

$M \in \mathcal{U}_{(p 3)}^{4}$

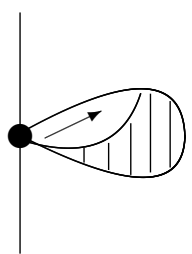

$M \in \mathcal{U}_{(p 3)}^{5}$

Figure 1: Five types of maps which will induce a nonroot-vertex loop after splitting the root-vertex.

Remark. (1) Maps of type 1 (or 2) have their edge $e_{r}(M)$ (or $e_{P r}(M)$ ) the planar loop, here $P$ is the rotation of $M$ at the root-vertex; (2) Maps of the above types will not create nonroot-vertex after splitting the root-vertex. 
There are 5 cases corresponding to the dividing of $\mathcal{U}_{(p 3)}$ which must be handled.

Case 1. The maps of $\mathcal{U}_{(p 3)}^{1}$.

Notice that for a map of $\mathcal{U}_{(p 3)}^{1}$, two things will definitely happen: the number of nonroot-vertex loops will increase and the number of nonroot-cut-vertices will not change after splitting the root-vertex. It is easy to see that the contributions of $\mathcal{U}_{p 3}^{1}$ to $\mathcal{U}_{p}$ is

$$
f_{p 3}^{1}=t y^{2} z f_{p}
$$

Case 2. The maps of $\mathcal{U}_{(p 3)}^{2}$.

Under this condition, the edge $e_{P r}(M)$ is always a planar loop while the root-edge $e_{r}(M)$ is a loop which will create a nonroot-vertex loop. This situation is very similar to the previous case except that $e_{r}(M)$ may be an essential loop. Thus, we find that the enumerating function of $\mathcal{U}_{p 3}^{2}$ is exactly equal to $f_{p 3}^{1}$, i.e.,

$$
f_{p 3}^{2}=t y^{2} f_{p}
$$

\section{Case 3. The maps of $\mathcal{U}_{(p 3)}^{3}$.}

Similar to what we have done in Cases 1 and 2 , one may partition the set $\mathcal{U}_{(p 3)}^{3}$ into two parts as

$$
\mathcal{U}_{(p 3)}^{3}=\mathcal{U}_{(p 3)}^{31}+\mathcal{U}_{(p 3)}^{32},
$$

where $\mathcal{U}_{(p 3)}^{31}=\left\{M \mid\right.$ both $\mathrm{e}_{\mathrm{r}}(\mathrm{M})$ and $\mathrm{e}_{\mathrm{P}^{2} \mathrm{r}}(\mathrm{M})$ are loops $\}$.

Hence maps in $\mathcal{U}_{(p 3)}^{31}$ have the following structure in the left side of Figure 2.
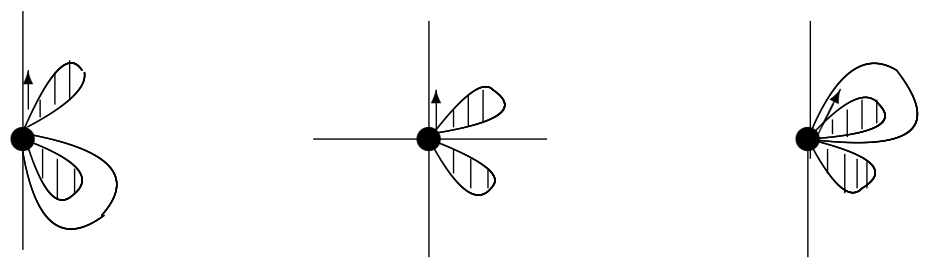

Figure 2: Three types of maps which will create a pair of multi-edges after splitting the root-vertex, where the shadowed regions represent planar maps.

Thus, the enumerating function of $\mathcal{U}_{p 3}^{3}$ is

$$
f_{p 3}^{3}=t y^{2}(f-1) .
$$

Case 4. The maps of $\mathcal{U}_{(p 3)}^{4}$.

As we have reasoned previously, we partition the set $\mathcal{U}_{(p 3)}^{4}$ into two parts as

$$
\mathcal{U}_{(p 3)}^{4}=\mathcal{U}_{(p 3)}^{41}+\mathcal{U}_{(p 3)}^{42},
$$


where $\mathcal{U}_{(p 3)}^{41}=\left\{M \mid\right.$ both $\mathrm{e}_{\mathrm{r}}(\mathrm{M})$ and $\mathrm{e}_{\mathrm{Pr}}(\mathrm{M})$ are crossing essential loops $\}$ and maps of $\mathcal{U}_{(p 3)}^{41}$ have a configuration as shown in the center of Figure 2. Therefore the contribution of $\mathcal{U}_{(p 3)}^{41}$ is $f_{(p 3)}^{41}=x^{4} y^{2} z f^{2}$ which implies

$$
f_{p 3}^{4}=t y^{2}(f-1)
$$

\section{Case 5. The maps of $\mathcal{U}_{(p 3)}^{5}$.}

Also the set $\mathcal{U}_{(p 3)}^{5}$ should be divided in a way of

$$
\mathcal{U}_{(p 3)}^{5}=\mathcal{U}_{(p 3)}^{51}+\mathcal{U}_{(p 3)}^{52},
$$

where $\mathcal{U}_{(p 3)}^{51}=\left\{M \mid e_{r}(M)\right.$ and $\mathrm{e}_{\mathrm{Pr}}(\mathrm{M})$ are two types of loops $\}$.

It is clear that the two edges $e_{r}(M)$ and $e_{P r}(M)$ are in a position as in the right side of Figure 2. Now one may readily see that the enumerating function of $\mathcal{U}_{p 3}^{5}$ is

$$
f_{p 3}^{5}=t y^{2}(f-1)
$$

\section{Case 6. The maps of $\mathcal{U}_{(p 3)}^{6}$.}

Before investigating the contribution of $\mathcal{U}_{(p 3)}^{6}$, we have to state some basic facts.

Fact 1. For a map $M$ of $\mathcal{U}_{(p 3)}^{6}$, splitting the root-vertex will not create nonroot-vertex loops but may increase the number of nonroot-cut-vertices.

Consequently, we have to divide the set $\mathcal{U}_{(p 3)}^{6}$ into two parts as

$$
\mathcal{U}_{(p 3)}^{6}=\mathcal{U}_{(p 3)}^{61}+\mathcal{U}_{(p 3)}^{62},
$$

where $M \in \mathcal{U}_{(p 3)}^{61} \Longleftrightarrow$ splitting the root-vertex will create a nonroot-cut-vertex.

Next we study the structures of the maps in $\mathcal{U}_{(p 3)}^{61}$. Since the splitting-procedure defined previously will create a nonroot-cut-vertex, say $v_{r}^{\prime \prime}$, we conclude that $v_{r}^{\prime \prime}$ will connect a map other than the vertex-map (i.e., the map consists of a single vertex) whose root-valency is 2 . Since there are two types of such 2 -valent maps attached to the vertex $v_{r}^{\prime \prime}$, the set $\mathcal{U}_{(p 3)}^{61}$ should be partitioned into three parts as

$$
\mathcal{U}_{(p 3)}^{61}=\mathcal{U}_{(A)}+\mathcal{U}_{(B)}+\mathcal{U}_{(C)}
$$

where maps of those types are depicted in Figure 3.

Lemma 3. The set $\mathcal{U}_{(A)}$ is a composition of two types of maps, one is planar while the other a nonplanar map, i.e.,

$$
\mathcal{U}_{(A)}=\left(\mathcal{U}_{p}(2)-L_{p}\right) \odot(\mathcal{U}-\vartheta)+(\mathcal{U}(2)-L) \odot \mathcal{U}_{p}
$$

where $\mathcal{U}_{p}(2)$ and $\mathcal{U}(2)$ are, respectively, the set of maps of $\mathcal{U}_{p}$ and $\mathcal{U}$ whose root-valencies are all 2 . Meanwhile, $L_{p}$ and $L$ are, respectively, the loop map on the projective plane and the sphere. 


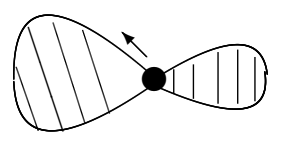

$M \in \mathcal{U}_{(A)}$

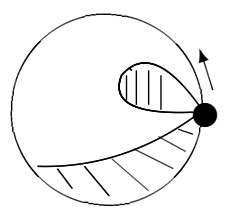

$M \in \mathcal{U}_{(B)}$

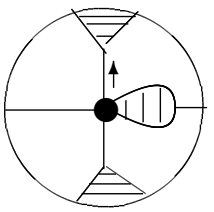

$M \in \mathcal{U}_{(C)}$

Figure 3: Three types of maps in $\mathcal{U}_{(p 3)}^{61}$ which will create a nonroot-cut-vertex after splitting the root-vertex.

Furthermore, by considering the embedding ways of the 2-valent maps attached to the nonroot-cut-vertices, the maps of $\mathcal{U}_{(A)}$ are classified into two types of maps as defined in Figure 4 , where the shadowed regions are either in $\mathcal{U}_{p}(2) \cup \mathcal{U}(2)$ or $\mathcal{U} \cup \mathcal{U}_{p}$.

Hence, the enumerating function of $\mathcal{U}_{(A)}$ is

$$
f_{(A)}=x^{2}\left(F_{p}(2)-y\right)(f-1)+x^{2}(F(2)-y z) f_{p},
$$

where $F_{p}(2)$ and $F(2)$ are, respectively, the enumerating function of $\mathcal{U}_{p}(2)$ and $\mathcal{U}(2)$.

It is clear that the three shadowed regions in the maps in the left side of Figure 5 are, respectively, two planar maps and a nonplanar one. Thus, the contribution of such types of maps is $x^{4} y z\left(F_{p}(2)-y\right) f^{2}$ and hence the other types of maps of $\left(\mathcal{U}_{p}(2)-L_{p}\right) \odot(\mathcal{U}-\vartheta)$ will contribute

$$
x^{2}\left(F_{p}(2)-y\right)(f-1)-x^{4} y z\left(F_{p}(2)-y\right) f^{2} .
$$

So we have

Lemma 4. The contribution of $\left(\mathcal{U}_{p}(2)-L_{p}\right) \odot(\mathcal{U}-\vartheta)$ to $\mathcal{U}_{p}$ is $w y\left(F_{p}(2)-y\right)(f-1)$.
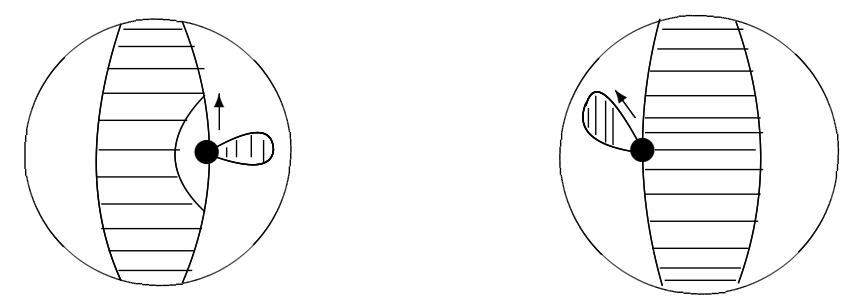

Figure 4: Two types of maps which will create a nonroot-cut-vertex after splitting the root-vertex.

We now turn to the second types of maps in Lemma 3. Unlike its counterpart, the set $(\mathcal{U}(2)-L) \odot \mathcal{U}_{p}$ consists of three types of maps among which two are defined, respectively, in the center and right parts of Figure 5.

It is easy to see that those two types of maps stated above will, respectively, contribute

$$
x^{4} y(F(2)-y z) \frac{\partial(x f)}{\partial x} \quad \text { and } \quad x^{4} y z(F(2)-y z) f_{p}
$$



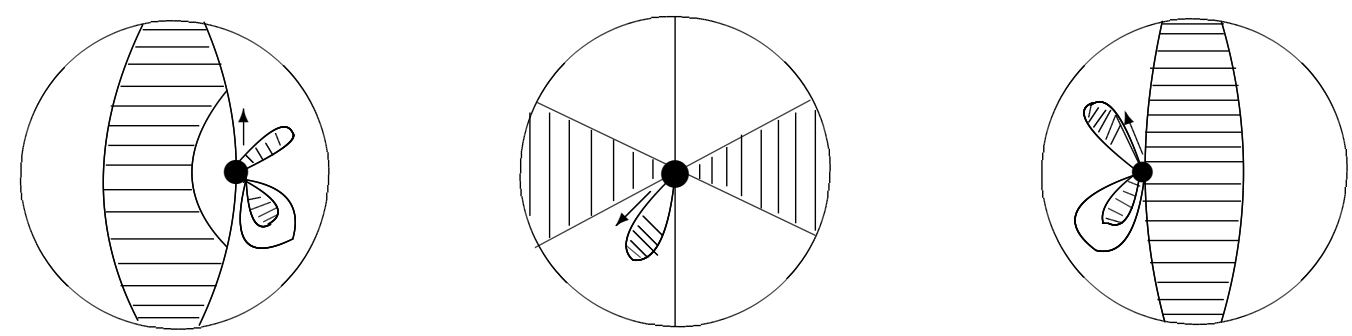

Figure 5: Three types of maps which will create a nonroot-cut-vertex after splitting the root-vertex.

to $\mathcal{U}_{(B)}$. Thus, we may obtain the following

Lemma 5. The contribution of $(\mathcal{U}(2)-L) \odot \mathcal{U}_{p}$ to $\mathcal{U}_{p}$ is $\frac{y w}{x^{2}}\left(x^{2}(F(2)-y z) f_{p}\right)$.

Now we begin to handle the set $\mathcal{U}_{(B)}$. Similar to Lemma 3 , we may have

Lemma 6. The set $\mathcal{U}_{(B)}$ is the composition of two types of maps, one is a planar map while the other is nonplanar, i.e.,

$$
\mathcal{U}_{(B)}=\left(\mathcal{U}_{p}(2)-L_{p}\right) \otimes(\mathcal{U}-\vartheta)+(\mathcal{U}(2)-L) \otimes \mathcal{U}_{p}
$$

where the operation " $\otimes$ " of maps is defined in Figure 6.
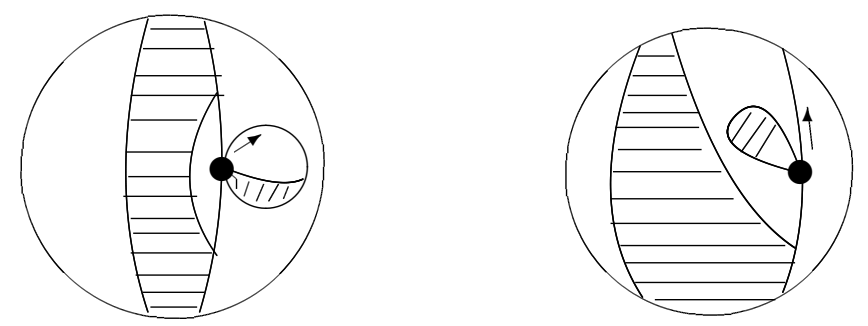

Figure 6: Two types of maps of $\mathcal{U}_{(B)}$ which will create a nonroot-cut-vertex after splitting the root-vertex.

After an analogous procedure in our proofs of Lemmas 4 and 6, we obtain the following

Lemma 7. The contribution of $\left(\mathcal{U}_{p}(2)-L_{p}\right) \otimes(\mathcal{U}-\vartheta)$ to $\mathcal{U}_{p}$ is $\frac{y w}{x^{2}}\left(x^{2}\left(F_{p}(2)-y\right)(f-1)\right)$.

Lemma 8. The contribution of $(\mathcal{U}(2)-L) \otimes \mathcal{U}_{p}$ to $\mathcal{U}_{p}$ is $\frac{y w}{x^{2}}\left(x^{2}(F(2)-y z) f_{p}\right)$.

Similar to what we have reasoned in Lemmas $4,5,7$ and 8 , the set $\mathcal{U}_{(C)}$ should be partitioned into two types of maps according to whether the root-vertex splitting-procedure will create a pair of multi-edges or not. The first kind of maps has a configuration in Figure 7, where the shadowed region represented by a perpendicular essential circuit is a map of $\mathcal{U}(2)-L$ embedded in $N_{1}$, the projective plane, while the other two indicate planar maps.

Lemma 9. The contribution of $\mathcal{U}_{(C)}$ to $\mathcal{U}_{p}$ is $\frac{y w}{x^{2}}\left\{\frac{x^{2}}{z}(F(2)-y z)(f-1)\right\}$. 


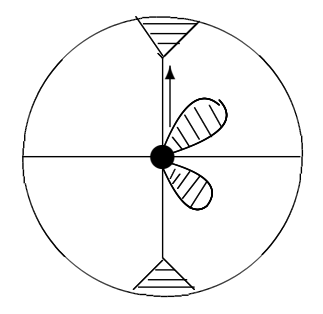

Figure 7: A type of maps of $\mathcal{U}_{(C)}$ which will create a nonroot-cut-vertex after splitting the root-vertex.

Now, we begin to establish the main result of this section. By (1) and (2), we have

$$
f_{p}=2 x^{2} y z f f_{p}+x^{2} y \frac{\partial(x f)}{\partial x}+f_{p 3},
$$

where

$$
f_{p 3}=\frac{y}{x^{2}}\left\{f_{p}-x^{2} F_{p}(2)\right\}+\sum_{i=1}^{5}\left(f_{p 3}^{i}-\frac{y}{x^{2}} f_{(p 3)}^{i}\right)+\left(f_{p 3}^{61}-\frac{y}{x^{2}} f_{(p 3)}^{61}\right)+\left(f_{p 3}^{62}-\frac{y}{x^{2}} f_{(p 3)}^{62}\right) .
$$

By equations (3)-(8),

$$
\begin{aligned}
& f_{p 3}^{1}-\frac{y}{x^{2}} f_{(p 3)}^{1}=y^{2} z(t-1) f_{p}, \\
& f_{p 3}^{2}-\frac{y}{x^{2}} f_{(p 3)}^{2}=y^{2} z(t-1) f_{p}, \\
& f_{p 3}^{3}-\frac{y}{x^{2}} f_{(p 3)}^{3}=(t-1) y^{2}(f-1), \\
& f_{p 3}^{4}-\frac{y}{x^{2}} f_{(p 3)}^{4}=(t-1) y^{2}(f-1), \\
& f_{p 3}^{5}-\frac{y}{x^{2}} f_{(p 3)}^{5}=(t-1) y^{2}(f-1), \\
& f_{p 3}^{61}-\frac{y}{x^{2}} f_{(p 3)}^{61}=\left\{f_{A}-\frac{y}{x^{2}} f_{(A)}\right\}+\left\{f_{B}-\frac{y}{x^{2}} f_{(B)}\right\}+\left\{f_{C}-\frac{y}{x^{2}} f_{(C)}\right\}, \\
& f_{A}-\frac{y}{x^{2}} f_{(A)}=(w-1) y\left(F_{p}(2)-y\right)(f-1)+(w-1) y(F(2)-y z) f_{p} \text {, } \\
& f_{B}-\frac{y}{x^{2}} f_{(B)}=(w-1) y\left(F_{p}(2)-y\right)(f-1)+(w-1) y(F(2)-y z) f_{p} \text {, } \\
& f_{C}-\frac{y}{x^{2}} f_{(C)}=\frac{(w-1) y}{z}(F(2)-y z)(f-1) \text {. }
\end{aligned}
$$

By the principle of Inclusion-Exclusion, the contribution of $f_{(p 3)}^{62}$ is

$$
f_{(p 3)}^{62}=\sum_{i=1}^{3}\left|R_{i}\right|-\sum_{1 \leqslant i<j \leqslant 3}\left|R_{i} \cap R_{j}\right|+\left|R_{1} \cap R_{2} \cap R_{3}\right|,
$$


where $|A|$ is used to denote the contribution of $A$ and

$$
\begin{aligned}
& R_{1}=\left\{M \mid e_{r}(M) \text { is a loop }\right\}, \\
& R_{2}=\left\{M \mid e_{P r}(M) \text { is a loop }\right\}, \\
& R_{3}=\left\{M \mid e_{P^{2} r}(M) \text { is a loop }\right\} .
\end{aligned}
$$

One may foresee from the possible embedding ways of loops on $N_{1}$ that the calculations of $f_{(p 3)}^{62}$ is very complicated. But its value is irrelevant for our further calculations since

$$
f_{p 3}^{62}=\frac{y}{x^{2}} f_{(p 3)}^{62}
$$

By substituting (10) and the above expressions into (9), we get our first main result:

Theorem A. The enumerating function of rooted (2-connected) near-4-regular maps on the projective plane satisfies the following equation:

$$
\begin{aligned}
f_{p}= & 2 x^{2} y z f f_{p}+x^{2} y \frac{\partial(x f)}{\partial x}+2 y^{2} z(t-1) f_{p}+3(t-1) y^{2}(f-1) \\
& +2(w-1) y\left(F_{p}(2)-y\right)(f-1)+2(w-1) y(F(2)-y z) f_{p} \\
& +\frac{(w-1) y}{z}(F(2)-y z)(f-1) .
\end{aligned}
$$

Remark. We have shown in [29] that the function $f$ defined in Theorem $\mathrm{A}$ is also algebraic, i.e., satisfying a polynomial equation, i.e.,

$$
a f^{2}+b f+c=0
$$

in which the coefficients of $f^{0}, f$ and $f^{2}$ can be expressed as

$$
\begin{aligned}
& a=x^{4} y z \\
& b=y-x^{2}+2(t-1) y^{2} z x^{2}+2 x^{2} y(w-1)\left(F_{2}-y z\right), \\
& c=x^{2}-y-x^{2} y F_{2}-2(t-1) x^{2} y^{2} z-2 x^{2} y(w-1)\left(F_{2}-y z\right) .
\end{aligned}
$$

If we rearrange the items of the equation in Theorem $\mathrm{A}$, then the function $f_{p}$ can be expressed as the functions of $f, F(2)$ and $F_{p}(2)$, i.e.,

$$
\begin{aligned}
-\sqrt{\triangle} f_{p}= & x^{4} y \frac{\partial(x f)}{\partial x}+3(t-1) x^{2} y^{2}(f-1)+2(w-1) x^{2} y\left(F_{p}(2)-y\right)(f-1) \\
& +\frac{(w-1) y x^{2}}{z}(F(2)-y z)(f-1)-x^{2} y F_{p}(2),
\end{aligned}
$$

where $\triangle$ is the discriminant of $(11)$, i.e.,

$$
\begin{aligned}
-\sqrt{\triangle}= & x^{2}-2 x^{4} y z f-y-4 x^{4} y^{3} z t(q-1) f-2 y t(t-1) x^{2} y z \\
& -2(w-1) x^{2} y(F(2)-y z) .
\end{aligned}
$$




\section{Calculations}

In this section we shall deal with various types of rooted (near-) 4-regular on the projective plane. Now the equation in Theorem A becomes

$$
\begin{aligned}
-\sqrt{\triangle} f_{p}= & x^{4} y \frac{\partial(x f)}{\partial x}+3(t-1) x^{2} y^{2}(f-1)+2(w-1) x^{2} y\left(F_{p}(2)-y\right)(f-1) \\
& +\frac{(w-1) y x^{2}}{z}(F(2)-y z)(f-1)-x^{2} y F_{p}(2),
\end{aligned}
$$

where

$$
-\sqrt{\triangle}=x^{2}-2 x^{4} y z f-y-2(t-1) x^{2} y^{2} z-2(w-1) x^{2} y z(F(2)-y z) .
$$

Now, we are in a position of implying the famous double-root method developed by Brown [8] since the most important part of $f_{p}$, the discriminant of (11), has become a quadratic polynomial of $x^{2}$. Let $x^{2}=\eta$ denote a double root of $\triangle$. Then we may obtain a system of equations:

$$
\begin{aligned}
& \eta-2 \eta^{2} y z f-y-2(t-1) \eta y^{2} z-2(w-1) \eta y(F(2)-y z)=0, \\
& x^{2} \frac{\partial(x f)}{\partial x}+3(t-1) y(f-1)+2(w-1)\left(F_{p}(2)-y\right)(f-1) \\
& +\frac{(w-1)}{z}(F(2)-y z)(f-1)-F_{p}(2)=0 .
\end{aligned}
$$

Let $t=w=1$. Then we get general rooted maps on $N_{1}$ and a very simple expression for $F_{p}(2)$ :

$$
F_{p}(2)=\left.\left\{x^{2} \frac{\partial(x f)}{\partial x}\right\}\right|_{x^{2}=\eta},
$$

which will imply the following known result:

Corollary 1 ([25]). The number of rooted 4-regular maps (which may have loops) on the projective plane with $2 p-2$ edges is

$$
\sum_{\substack{m \geqslant 2 \\
n \geqslant 0 \\
m+n \leqslant p}} \frac{2^{n+1} 3^{p-m-n}(m+n)}{m p}\left(\begin{array}{c}
2 m-2 \\
m-1
\end{array}\right)\left(\begin{array}{c}
m+n-2 \\
m-2
\end{array}\right)\left(\begin{array}{c}
2 p-m-n-1 \\
p-1
\end{array}\right)+\frac{3^{p-1}}{p}\left(\begin{array}{c}
2 p-2 \\
p-1
\end{array}\right) .
$$

Corollary 2 ([14, 26]). The enumerating function of rooted one-faced maps on the projective plane is

$$
\frac{1}{\sqrt{1-4 x^{2}}} \sum_{m \geqslant 1}\left(\begin{array}{c}
2 m-1 \\
m
\end{array}\right) x^{2 m}
$$

If we consider the case of loopless maps (i.e., $t=0$ ), then we have the following 
Corollary 3 ([26]). The enumerating function of rooted loopless 4-regular maps on the projective plane is

$$
\sum_{m \geqslant 2}\left(A_{m}+\frac{3}{2} Y_{m-1}-3 X_{m-1}-X_{m}\right)\left(1-2 y^{2}\right) y^{2 m}-\sum_{m \geqslant 2} 3 B_{m-1} y^{2 m}
$$

in which

$$
\begin{aligned}
A_{m}= & \sum_{\substack{l, p \geqslant 0 \\
k \geqslant 2}} \sum_{\substack{s+p=m+1 \\
s \geqslant k+2}} \frac{(-1)^{p} 2^{l+p+1} 3^{s-k-l}(k+l)}{k \times s}\left(\begin{array}{c}
2 k-2 \\
k-1
\end{array}\right) \\
& \times\left(\begin{array}{c}
k+l-2 \\
l
\end{array}\right)\left(\begin{array}{c}
2 s-k-l-1 \\
s-1
\end{array}\right)\left(\begin{array}{c}
2 s+p-2 \\
p
\end{array}\right), \\
B_{m}= & \sum_{l+n=m} \frac{(-1)^{l} 2^{l} 3^{n}}{(n+1)(2 n+1)}\left(\begin{array}{c}
2 n+2 \\
n
\end{array}\right)\left(\begin{array}{c}
n+m \\
l
\end{array}\right), \\
X_{m}= & \sum_{l+n=m} \frac{(-1)^{l} 2^{l+1} 3^{n}}{n+1}\left(\begin{array}{c}
2 n \\
n
\end{array}\right)\left(\begin{array}{c}
n+m \\
l
\end{array}\right), \\
Y_{m}= & \sum_{l+n=m} \frac{(-1)^{l} 2^{l+3} 3^{n}}{n+2}\left(\begin{array}{c}
2 n+1 \\
n
\end{array}\right)\left(\begin{array}{c}
n+m \\
l
\end{array}\right) .
\end{aligned}
$$

Next, we start to investigate the number of 2-connected 4-regular maps on the projective plane. We first consider general 2-connected maps (which may have loops).

\section{Case 1. General 2-connected 4-regular maps on $N_{1}$.}

Let $t=y=1$ and $w=0$. Then all the cut-vertices disappear except for those on the root-vertices by our definition. Furthermore, let $x^{2}=\eta$ be a double root of $\triangle$, the discriminant of (11). Then all the functions and variables in this case are handled under those conditions unless special statements are given. Now the function $F_{p}(2)$ can be expressed in terms of $f$ and $F(2)$, i.e.,

$$
(2 f-1) F_{p}(2)=\left.\left\{x^{2} \frac{\partial(x f)}{\partial x}-\frac{F}{z}(f-1)+2(f-1)\right\}\right|_{x^{2}=\eta},
$$

where $F=F(2)-z$.

In order to determine the function $F_{p}(2)$ completely, we have to state some facts as our step-stones.

Fact 2 ([29]). The function $F$ may be expressed in terms of $\eta$ and $z$, i.e.,

$$
F=\frac{1-\eta+2 \eta^{3} z(1-z)}{2 \eta\left(1-\eta^{2} z\right)}
$$

in which

$$
\eta=1+\frac{z}{2} \phi(\eta, z), \quad \phi(\eta, z)=\frac{\eta^{2}(1+\eta-2 \eta z)^{2}}{(1+2 \eta z)\left(1-\eta^{2} z\right)}
$$


Since the function $f$ is algebraic, after some manipulations of the system of equations (12) we may find the following

Fact 3. The functions $F$ and $f$ are related in such a way that

$$
\eta-2 \eta^{2} z f-1+2 \eta F=0, \quad \eta \frac{\partial(x f)}{\partial x}=\frac{1-\sqrt{1-v \eta}}{\eta z}-\eta f,
$$

where $f=\frac{1}{1-\eta^{2} z f}$.

The Lagrangian inversion formula stated above is vital in our simplification of the parametrical expression of $F_{p}(2)$. In fact, another inversion formula cited in Fact 2 may also be expressed in terms of $f$, i.e.,

$$
\eta=1+\frac{z}{2} \phi(\eta, z), \quad \phi(\eta, z)=\eta f\left\{1+\frac{\eta f}{1-f}\right\} .
$$

Based on Facts 2 and 3, the function $F_{p}(2)$ can be expressed by $\eta$ and $f$ elegantly, i.e.,

Fact 4. The enumerating function of rooted 2-valent near-4-regular maps (which may have loops) without cut-vertices on the projective plane is

$$
(2 f-1) F_{p}(2)=\frac{1-\sqrt{1-v \eta}}{\eta z}+\frac{\eta(\eta-3)}{2} f^{2}+2(f-1),
$$

in which $v \eta=4 \eta^{2} z f$.

Although $F_{p}(2)$ presents a formula to count a type of rooted 2-connected 4-regular maps on $N_{1}$, we may readily see from the definition that if loops are permitted, then the enumerating function for rooted 2-connected 4-regular maps on $N_{1}$ depends on both $F_{p}(2)$ and the same type of maps on the plane. In fact, rooted 2-connected 4-regular maps on $N_{1}$ consist of 5 types of maps which are depicted in Figure 8.
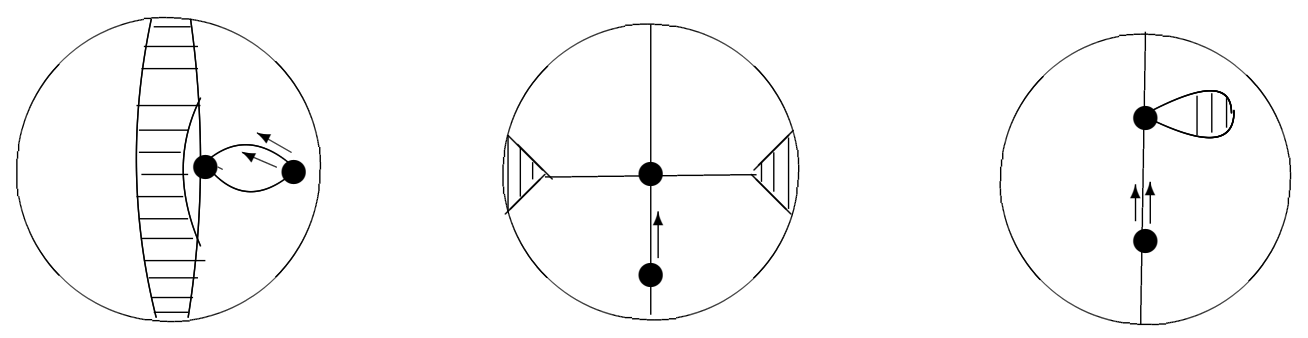

Figure 8: Five types of 2-valent maps which will result in five kinds of 4-regular maps on $N_{1}$.

Fact 5. The enumerating function of rooted 2-connected 4-regular maps on $N_{1}$ can be expressed as

$$
F_{p}(4)=(1+2 z)\left(F_{p}(2)-1\right)+3 F,
$$


where $F_{p}(2)$ and $F$ are defined in Facts 2 and 4.

By using Lagrangian inversion (for a reference, one may see [16]) for these in (13) and Fact 3, the two functions may be expanded into a power series of the variable $z$, i.e.,

Theorem B. The enumerating function of rooted 4-regular maps (which may have loops) without cut-vertices on the projective plane is

$$
\begin{aligned}
& F_{p}(4)=\sum_{\substack{k \geqslant 0 \\
n \geqslant k}} \frac{2^{k} k}{n}\left(\begin{array}{c}
2 n-k-1 \\
n-1
\end{array}\right)(1+2 z) z^{n-k} \eta^{2(n-k)} \\
& +\sum_{\substack{k \geqslant 0 \\
n \geqslant k+2}} \frac{2^{k-1}(k+2)}{n}\left(\begin{array}{c}
2 n-k-3 \\
n-1
\end{array}\right)(1+2 z) z^{n-k-2}(\eta-3) \eta^{2(n-k-2)+1} \\
& +\sum_{\substack{k \geqslant 1 \\
l \geqslant 0}} \sum_{n \geqslant k+l} \frac{2^{l+1}(k+l)}{k n}\left(\begin{array}{c}
2 k-2 \\
k-1
\end{array}\right)\left(\begin{array}{c}
2 n-k-l-1 \\
n-1
\end{array}\right)(1+2 z) z^{n-l-1} \eta^{2(n-l)-1} \\
& +3 \sum_{\substack{m \geqslant 2 \\
n \geqslant m}} \frac{m}{2 n}\left(\begin{array}{c}
2 n-m-1 \\
n-1
\end{array}\right) z^{n-m+1}(\eta-1) \eta^{2(n-m)+1}+3 \sum_{n \geqslant 1} \frac{1}{n}\left(\begin{array}{c}
2 n-2 \\
n-1
\end{array}\right) z^{n} \eta^{2 n-1},
\end{aligned}
$$

where

$$
\begin{aligned}
\eta^{s}= & \sum_{m \geqslant 1} \sum_{p=0}^{m} \sum_{\substack{q \geqslant 0 \\
n \geqslant m+p+q}} \frac{s(m+p+q)}{m ! n}\left(\begin{array}{c}
2 n-m-p-q-1 \\
n-1
\end{array}\right) \\
& \times\left(\begin{array}{c}
p+q-1 \\
p-1
\end{array}\right) D_{\eta=1}^{(m-1)}\left\{\eta^{2 n-m-p-2 q}\right\} z^{n-q},
\end{aligned}
$$

in which the differential operator " $D_{\eta=1}^{(m-1)}$ " is defined as

$$
D_{x=a}^{(m-1)} H=\left.\frac{\partial^{m-1} H}{\partial x}\right|_{x=a} .
$$

By using an algebraic symbolic system, say Maple, the first fewer coefficients of the function $F_{p}(4)$ may be calculated:

$$
\begin{aligned}
F_{p}(4)= & 5 z+38 z^{2}+199 z^{3}+1466 z^{4}+12365 z^{5}+109700 z^{6} \\
& +1003929 z^{7}+9404402 z^{8}+\cdots
\end{aligned}
$$

One may see that the low values of maps coincide with our calculation. For instance, there are 38 distinct rooted 2-connected 4-regular maps with 4 edges on the projective plane which are determined by the maps shown in Figure 9.

The contributions of the 6 maps to the rooted maps are, respectively, listed in the following table:

\begin{tabular}{|c|c|c|c|c|c|}
\hline$M_{1}$ & $M_{2}$ & $M_{3}$ & $M_{4}$ & $M_{5}$ & $M_{6}$ \\
\hline 8 & 8 & 8 & 2 & 4 & 8 \\
\hline
\end{tabular}



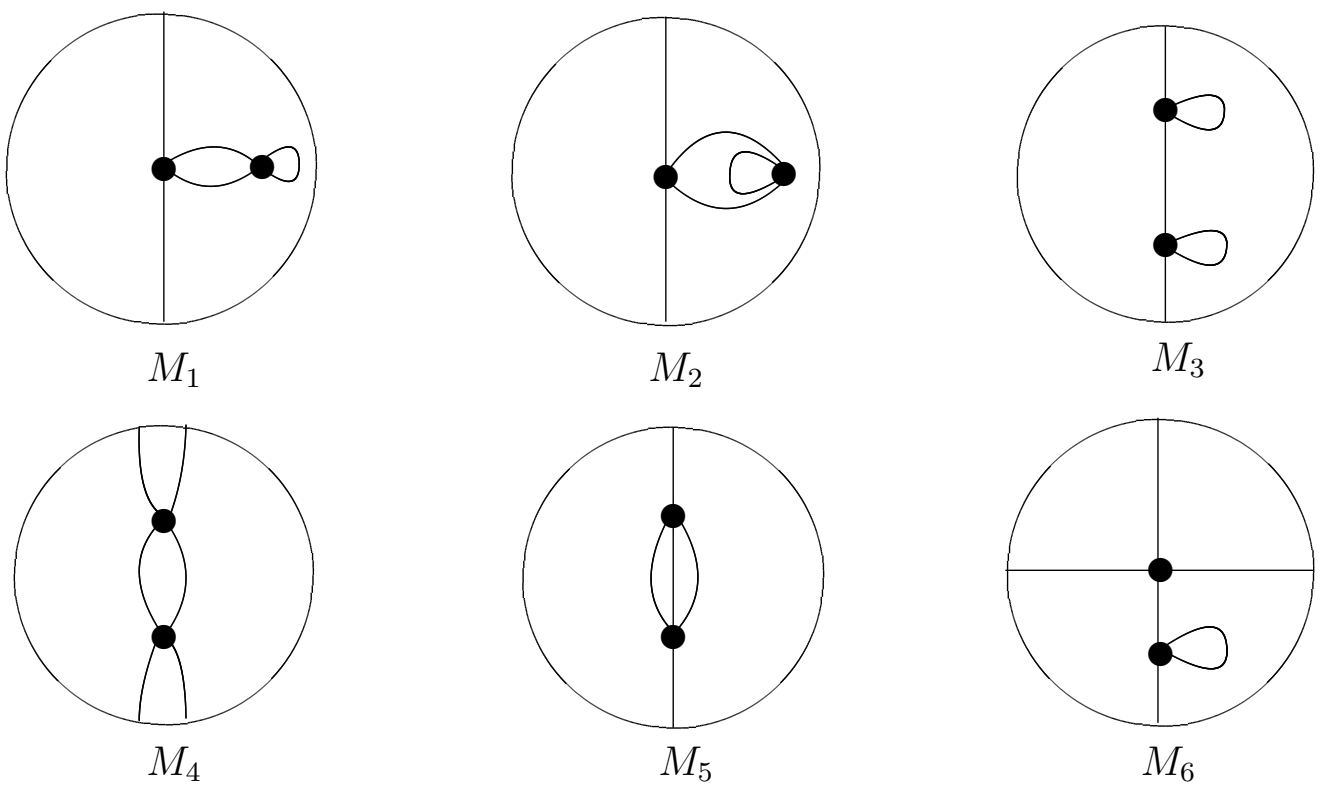

Figure 9: Six unrooted maps on $N_{1}$ which will determine 38 distinct rooted maps.

Remark. The results stated previously permit the existence of loops and maps there are slightly different from what defined by Tutte [30] where a 2-connected graph (map) contains no loops. This is just the situation we shall handle next.

\section{Case II. 2-connected 4-regular maps without loops.}

Let $y=1$ and $t=w=0$. Then all the loops and cut-vertices are eliminated except for those on the root-vertices. Still let $x^{2}=\eta$ be a double root of the discriminant of (11). Then all the functions and variables appearing in this case are all under those restrictions unless special statements are given. Similar to what we have stated previously, we have Fact 6 ([29]). The function $F$ may be expressed as a function of $\eta$ and $z$, i.e.,

$$
\begin{aligned}
& F=\frac{1-\eta-2 \eta z+2 \eta^{3} z(1+z)}{2 \eta\left(1-\eta^{2} z\right)} \\
& \eta=1+z \phi(\eta, z), \quad \phi(\eta, z)=\eta^{2}\left\{\frac{(1+\eta)^{2}}{2\left(1-\eta^{2} z\right)}-2\right\}
\end{aligned}
$$

in which $F=F(2)-z$.

Fact 7. The function $F$ and $f$ are related in such a way that

$$
\eta-2 \eta^{2} z f-1+2 \eta z+2 \eta F=0, \quad \eta \frac{\partial(x f)}{\partial x}=\frac{1-\sqrt{1-v \eta}}{\eta z}-\eta f,
$$

where

$$
f=\frac{1}{1-\eta^{2} z f}
$$


Remark. There are several things striking us most: (1) The formula (16) is almost the same as the one for trees presented by Tutte [33] and this raises a natural question: Can we construct planar (near-)4-regular maps from trees? (2) Eliminating loops will not change some properties cited in the Facts 2 and 3, especially the formula in Fact 3 (which is the same as (16)). But from the results in [29] one may see that those two types of 2-connected 4-regular maps on the plane are indeed different in essentiality since we have shown there that their convergence radius are distinct. Instead, as our discussion goes on, one may see that such strict condition on maps (i.e., destroying loops) will make our calculations much more easier and simpler. Besides, the function $\phi(\eta, z)$ appearing in Fact 5 can be simply expressed as a function of $f$, i.e.,

$$
\begin{aligned}
\phi(\eta, z) & =\eta^{2}\left\{\frac{(1+\eta)^{2}}{2\left(1-\eta^{2} z\right)}-2\right\} \\
& =2 \eta^{2} f(f-1),
\end{aligned}
$$

which will lead to a simpler formula of $F_{p}(2)$.

Since the procedure is very much alike as we get in Theorem B, we omit the proofs and only state them here.

Theorem C. The enumerating function of 2-connected loopless rooted 4-regular maps on the projective plane is

$$
F_{p}(4)=\frac{1-\sqrt{1-v \eta}}{\eta z(2 f-1)}-\frac{\eta f}{2 f-1}-\frac{\eta^{3} z f^{3}(2-f)}{2 f-1}-1,
$$

in which $v \eta=4 \eta^{2} z f$.

After applying Lagrangian inversion for the formulae in Facts 4 and 5, we may expand $F_{p}(4)$ into a power series of $z$, i.e.,

$$
\begin{aligned}
F_{p}(4)= & \sum_{\substack{k \geqslant 0 \\
n \geqslant k+3}} \frac{2^{k} 3(k+3)}{n}\left(\begin{array}{c}
2 n-k-4 \\
n-1
\end{array}\right) z^{n-k-2} \eta^{2(n-k-2)+1} \\
& -\sum_{\substack{k \geqslant 0 \\
n \geqslant k+3}} \frac{2^{k}(k+3)}{n}\left(\begin{array}{c}
2 n-k-4 \\
n-1
\end{array}\right) z^{n-k-3} \eta^{2(n-k-3)+1} \\
& -\sum_{\substack{k \geqslant 2 \\
l \geqslant 0}} \sum_{n \geqslant k+l} \frac{2^{l+1}(k+l)}{k n}\left(\begin{array}{c}
2 k-2 \\
k-1
\end{array}\right)\left(\begin{array}{c}
2 n-k-l-1 \\
n-1
\end{array}\right) z^{n-l-1} \eta^{2(n-l)-1} \\
& -1,
\end{aligned}
$$

where for $s \geqslant 1$,

$$
\eta^{s}=1+\sum_{\substack{m \geqslant 1 \\
p \geqslant 0}} \sum_{q \geqslant 2 m+p} \frac{s(2 m+p)}{q(2 q+s)}\left(\begin{array}{c}
m+p-1 \\
p
\end{array}\right)\left(\begin{array}{c}
2 q-2 m-p-1 \\
q-1
\end{array}\right)\left(\begin{array}{c}
2 q+s \\
m
\end{array}\right) z^{q} .
$$


After some manipulations via an algebraic system such as Maple, the first fewer coefficients of the function $F_{p}(4)$ may be determined:

$$
F_{p}(4)=6 z^{2}+21 z^{3}+138 z^{4}+781 z^{5}+4836 z^{6}+30099 z^{7}+191698 z^{8}+\cdots
$$

One may compare the initial values of maps with the number presented by this formula and see that they are coincide with each other. For instance, there are 6 rooted loopless 4regular maps with 4 edges on $N_{1}$ as determined by the two maps $M_{4}$ and $M_{5}$ in Figure 10 . Furthermore, Figure 10 shows another group of unrooted maps which will induce 21 distinct rooted maps with 4 faces on $N_{1}$.
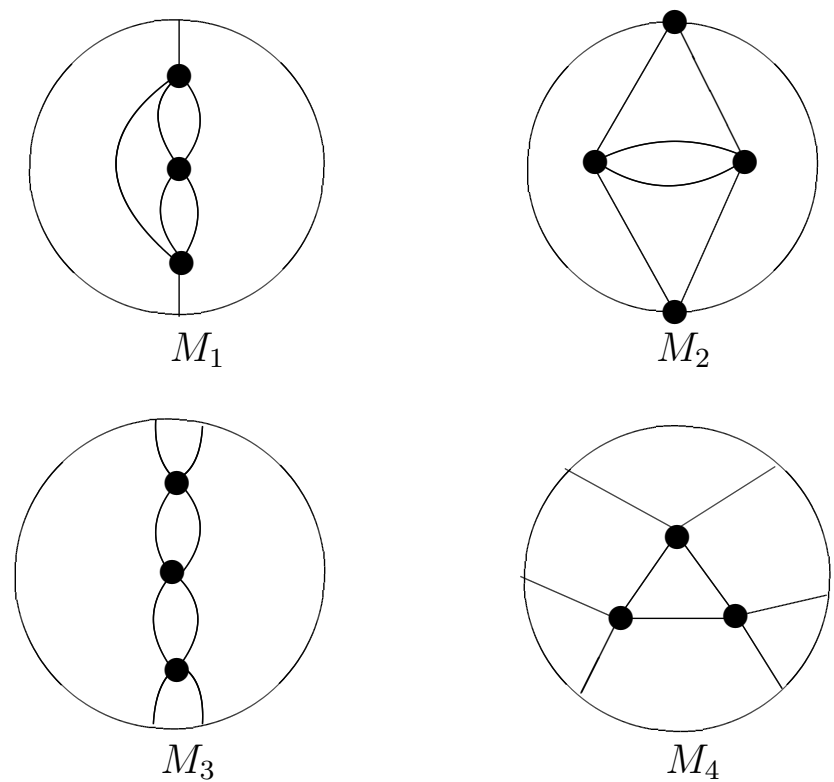

Figure 10: Four distinct embedding of the double graph $K_{3}^{2}$ on $N_{1}$ which will induce 21 rooted maps.

The contributions of the four maps to the rooted maps are, respectively, listed in the following table:

\begin{tabular}{|c|c|c|c|}
\hline$M_{1}$ & $M_{2}$ & $M_{3}$ & $M_{4}$ \\
\hline 12 & 6 & 2 & 1 \\
\hline
\end{tabular}

\section{Asymptotic evaluations}

In this section we concentrate on the approximate values of the number of the two types of maps obtained in the last section. Since applying of the Darboux's theorem [2, Theorem 4] needs some theory backgrounds, we have to state some basic facts before our evaluations.

Fact 8. Let $M$ be a class of infinite rooted maps with $M_{1}$ as its subset. Suppose that their enumerating functions may be expanded into power series and their respective convergence radiuses are $R$ and $R_{1}$. Then $R$ and $R_{1}$ are, respectively, the singularities of them. Furthermore, $R \leqslant R_{1}$. 
In the case of $y=t=w=1$, the function $F_{p}(2)$ has a very simple expression:

$$
z F_{p}(2)=1-\frac{3 \theta}{2}-(1-\theta) \sqrt{1-\frac{2 \theta}{1-\theta}}, \quad \theta=\frac{4 z}{2-3 \theta} .
$$

By a result in [2, Theorem 5] and Darboux's Theorem [2, Theorem 4], we have:

Fact $\mathbf{9}[\mathbf{1 5}]$. The convergence radius of the power series expansion with the number of inner faces as the parameter of the enumerating function for the rooted 4-regular maps on the projective plane is $\frac{1}{12}$ and consequently, the number of rooted 4-regular maps on the projective plane with $n$ inner faces is asymptotic to

$$
-\frac{C 12^{n}}{n^{\frac{5}{4}} \Gamma\left(-\frac{1}{4}\right)}
$$

where $C=\frac{\sqrt{6}}{3}$. Furthermore, any type of infinite rooted 4-regular maps on the projective plane must have its convergence radius, say $r$, of enumerating function satisfying

$$
\frac{1}{12} \leqslant r \leqslant 1
$$

We first consider the asymptotic evaluation of the number of rooted 2-connected 4regular maps (which may have loops) on $N_{1}$, i.e., $y=t=1$ and $w=0$. So all the discussions in this case are under those restrictions unless special statements are given. The expressions in Fact 2 may be rewritten as

$$
F=\frac{1-x+2 x^{3} z(1-z)}{2 x\left(1-x^{2} z\right)}, \quad x=1+\frac{z x^{2}(1+x-2 x z)^{2}}{2(1+2 x z)\left(1-x^{2} z\right)} .
$$

Remark. Here we use $x^{2}=x=\eta$ to denote the double root of (11) in convenience.

Since Darboux's Theorem needs the location of the singularity corresponding to the convergence radius of the enumerating function, we have to determine the convergence radius of $F_{p}(4)$ defined in Fact 5 . By definition of $F_{p}(4)$, one may see the following

Fact 10. The singularities of $F_{p}(4)$ satisfy either

(1) $2 f-1=0$,

(2) or $x=0$,

(3) or $v x=1$,

(4) or Equations in (20).

It is clear that (17) implies $x \neq 0$. If $2 f=1$, then by Fact 3 we have $x=1$ or $1+2 x z=0$ either will contradict (17). So the wanted singularities will be determined by $x v=1$ or equations of (17). The condition $v x=4 x^{2} z f$ together with (18) shows that the singularities of $F_{p}(4)$ will be induced by the following system of equations:

$$
\begin{aligned}
4(x-1)(1+2 x z) & =1+x-2 x z, \\
2 x^{2} z(x+1-2 x z) & =1-x^{2} z .
\end{aligned}
$$


The variable $z$ my be extracted from the above equations such that

$$
z=\frac{5-3 x}{2 x(4 x-3)}
$$

We now substitute it into an equation of (18) and arrive at another equation of $x$ :

$$
x(5-3 x)\left(4 x^{2}+6 x-\frac{9}{2}\right)-(4 x-3)^{2}=0 .
$$

By using Maple we may solve the equation by factoring the left side of (19) as

$$
(x-1)(x+2)\left(24 x^{2}-28 x-9\right)=0
$$

and find its zeros and the corresponding values of $z$ as listed in the following table:

\begin{tabular}{|c|c|c|c|c|}
\hline$x$ & 1 & -2 & $\frac{7+\sqrt{103}}{12}$ & $\frac{7-\sqrt{103}}{12}$ \\
\hline$z$ & 1 & $\frac{1}{4}$ & 0.0918035245 & 2.723004459 \\
\hline
\end{tabular}

Now, what left for us is to determine the wanted singularity corresponding to the convergence radius. The following fact is useful in our evaluations.

Fact 11. The convergence radius of the power series with the number of inner faces as the parameter for the rooted 2-connected 4-regular planar maps is 0.0918035245 . Furthermore, the convergence radius, say $r$, of the power series expansion of $F_{p}(4)$ must satisfy the following condition:

$$
\frac{1}{12} \leqslant r \leqslant 0.0918035245
$$

which shows that the convergence radius of power series of $F_{p}(4)$ is 0.0918035245 .

The proof of the first part of the fact may be found in [29]. The left side of the inequality follows from Fact 9. Since any rooted 2-connected 4-regular planar map may be re-embedded into $N_{1}$ by making a twist on its root-edge (the operation is shown in Figure 11), the set of rooted 2-connected 4-regular planar maps may be viewed as an infinite subset of $\mathcal{U}_{p}(4)$, the set of rooted 2-connected 4-regular maps on $N_{1}$. Thus, the rest part of the inequality follows from Fact 8 and the table below (20).

Let $F_{p}(4)=(1+2 z)\left(F_{p}(2)-1\right)+3 F$ be as defined in Fact 5 and $m=0.0918103549$ be the convergence radius of $F_{p}(4)$. In order to apply Darboux's method [2, Theorem 4], we should study the behavior of $x=x(z)$ near $m$. Since both $\frac{\partial^{2} H(x, z)}{\partial x^{2}}$ and $\frac{\partial H(x, z)}{\partial x}$ are not equal to zero (where the function $H(x, z)$ is defined by the Lagrangian inversion formula in $(17)), x=x(z)$ may be expanded into a power series of $\left(1-\frac{z}{m}\right)^{\frac{1}{2}}$ near $m$. Let

$$
\begin{aligned}
& x=a+b\left(1-\frac{z}{m}\right)^{\frac{1}{2}}+c\left(1-\frac{z}{m}\right)+d\left(1-\frac{z}{m}\right)^{\frac{3}{2}}, \\
& z=m-m\left(1-\frac{z}{m}\right) .
\end{aligned}
$$




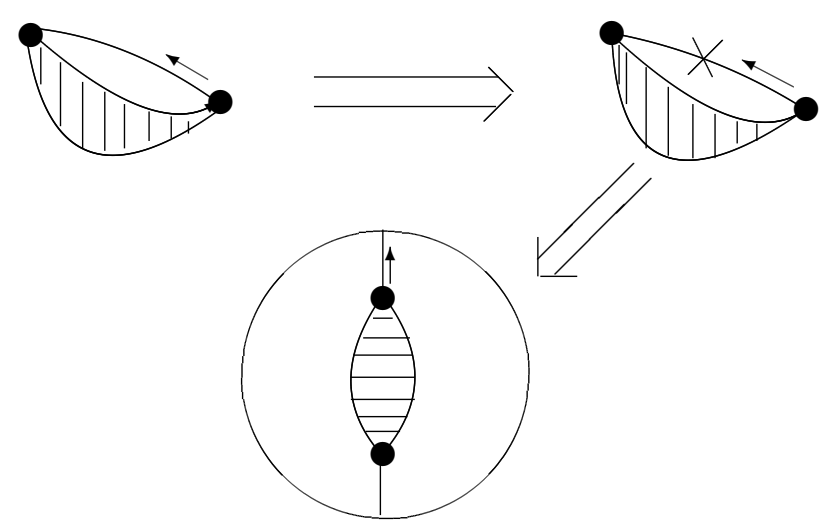

Figure 11: Re-embedding a rooted planar map into the projective plane by twisting the root-edge.

Substitute those into the equation system (18) and let the coefficients of $\left(1-\frac{z}{m}\right)$ and $\left(1-\frac{z}{m}\right)^{\frac{3}{2}}$ be zero. Further, by using a polynomial $24 a^{2}-28 a-9$ induced in (20) to reduce the orders of the corresponding polynomials appearing the identities obtained, we may find a group of relations such as

$$
\begin{aligned}
& a=\frac{7+\sqrt{103}}{12}, \quad b^{2}=\frac{\alpha}{\beta}, \\
& c=\frac{m\left(8 a^{3} m-12 m b^{2} a-2 a^{3}+5 a^{2}+6 b^{2} a-6 a-5 b^{2}\right)}{-6 m+12 m^{2} a^{2}-6 m a^{2}+10 m a+3}, \\
& d=\frac{b\left(3 a+5 a^{2} m-4 a^{3} m+4 a^{3} m^{2}+5 b^{2} m-6 a b^{2} m+12 a m^{2} b^{2}\right)}{a\left(-6 m+12 m^{2} a^{2}-6 m a^{2}+10 m a+3\right)},
\end{aligned}
$$

where

$$
\begin{aligned}
\alpha= & (1+2 m)\left(-559498752 a m+3077533008 a m^{2}+40310784 a\right. \\
& +50868841072 a m^{6}-63747912240 a m^{5}-10902385800 a m^{3} \\
& +26896643452 a m^{4}+692327412 m^{2}-81041472 m+7057741014 m^{4} \\
& \left.-2826562311 m^{3}-16728212988 m^{5}+13348368792 m^{6}\right), \\
\beta= & 432 m\left(279410720 a m^{6}+265248 a m-23432500 a m^{3}\right. \\
& -129887248 a m^{5}+172029608 a m^{4}+22625424 a m^{2} \\
& +279936 a+45176364 m^{4}+1242216 m-5852502 m^{3} \\
& \left.-155520+4298103 m^{2}+73304640 m^{6}-33897240 m^{5}\right) .
\end{aligned}
$$

Remark. We only need to expand $x(z)$ into the form of (21). If necessary, higher terms have to be introduced and hence more parameters such as $a, b, c, d$ will appear.

Now, we begin to investigate the asymptotic behavior of $F_{p}(2)$ near $m$. By (16)

$$
1-v x=2 \sqrt{1-4 x^{2} z}-1
$$


which implies that

$$
\sqrt{1-v x} \approx \sqrt{\frac{-8 a m b}{\sqrt{1-4 a^{2} m}}}\left(1-\frac{z}{m}\right)^{\frac{1}{4}}
$$

and therefore

$$
\frac{1-\sqrt{1-v x}}{x z(2 f-1)} \approx-0.5079632059\left(1-\frac{z}{m}\right)^{\frac{1}{4}} .
$$

By Darboux's method, the coefficient of $z^{n}$ of the power series of $F_{p}(2)$ is asymptotic to

$$
-0.5079632059 \frac{n^{-\frac{5}{4}} m^{n}}{\Gamma\left(-\frac{1}{4}\right)} .
$$

This together with the approximate values of the coefficients of $F$ obtained in [29] shows the following

Theorem D. The number of rooted 2-connected 4-regular maps on $N_{1}$ with $n$ inner faces is asymptotic to

$$
-\frac{C_{1}(1+2 m)}{n^{\frac{5}{4}} m^{n} \Gamma\left(-\frac{1}{4}\right)},
$$

where $\Gamma(x)$ is the gamma function and $m=0.0918103549, C_{1}=0.5079632059$.

Finally, we evaluate the asymptotic values of the number of rooted 2-connected 4regular maps without loops on $N_{1}$. All the discussions next are based on the conditions that $y=1$ and $t=w=0$. Furthermore, we still let $x^{2}=x=\eta$ denote a double root of (11) in convenience. Now expressions of (17) and the function $F$ may be rewritten as

$$
\begin{aligned}
& x=1+z x^{2}\left\{\frac{(1+x)^{2}}{2\left(1-x^{2} z\right)}-2\right\}=1+2 x^{2} z f(f-1), \\
& F=\frac{1-x-2 x z+2 x^{3} z(1+z)}{2 x\left(1-x^{2} z\right)}
\end{aligned}
$$

where $f$ satisfies $(16)$.

As we have reasoned previously, we should first locate the singularity which corresponds to the convergence radius of the function $F_{p}(4)$. Theorem $\mathrm{C}$ guarantee the following

Fact 12. The singularities of $F_{p}(4)$ satisfy either

(1) $2 f-1=0$,

(2) or $x=0$,

(3) or $v x=1$,

(4) or Equations in (22).

After a very similar procedure as we did in our proof of Fact 11, the singularity determined by the sources in Fact 12 which corresponds to the convergence radius of $F_{p}(4)$ is located at $\frac{27}{196}$, i.e., both of the enumerating functions of rooted 2-connected 4-regular maps without loops on the sphere and the projective plane have the same convergence 
radius. But as we will see that their asymptotic behaviors near $\frac{27}{196}$ are basically distinct. Also we notice that nearly all the discussions used in our proof of Theorem $D$ are still valid except for the corresponding parameters such as $a, b, c, d$ and $m$, we obtain the following

Theorem E. The number of rooted 2-connected 4-regular maps without loops on the projective plane with $n$ inner faces is asymptotic to

$$
-\frac{C_{3}}{n^{\frac{5}{4}} m^{n} \Gamma\left(-\frac{1}{4}\right)},
$$

where

$$
C_{3}=\frac{\sqrt{\frac{8 a b m}{\sqrt{1-4 a^{2} m}}}}{a m\left(1+\frac{-1+\sqrt{1-4 a^{2} m}}{a^{2} m}\right)},
$$

and

$$
a=\frac{7}{6}, \quad m=\frac{27}{196}, \quad b=\frac{7 \sqrt{18766662918506}}{61089624} .
$$

Acknowledgements The computation of the approximate resultants were carried with the aid of algebra system (Maple). The authors would like one of referees whose suggestion to ignore the effects of multi-edges greatly simplifies the case analysis and many of the expressions and consequently improves the presentation of the paper.

\section{References}

[1] D. Arquès, Relations fonctionelles et dénombermant des cartes pointées sur le tore, J. Combin. Theory Ser. B 43 (1987), 253-274.

[2] E.A. Bender, Asymptotic methods in enumerations, SIAM Rev. 16 (1974), 485-515.

[3] E.A. Bender, L.B. Richmond, A survey of the asymptotic behaviour of maps, $J$. Combin. Theory Ser. B 40 (1986), 297-329.

[4] E.A. Bender, E.R. Canfield, L.R. Richmond, The asymptotic number of rooted maps on a surface, J. Combin. Theory Ser. A 63 (1993), 318-329.

[5] E.A. Bender, E.R. Canfield, R.W. Robinson, The enumeration of maps on the torus and the projective plane, Canad. Math. Bull. 31 (1988), 257-271.

[6] E.A. Bender, E.A. Canfield, The number of rooted maps on an orientable surface, J. Combin. Theory Ser. B 53 (1991), 293-299.

[7] J.A. Bondy, U.S.R. Murty, Graph Theory with Applications, Macmilan Press Ltd, 1976.

[8] W.G. Brown, On the existence of square roots in certain rings of power series, Math. Annalen 158 (1965), 82-89.

[9] W.G. Brown, Enumeration of quadrangular dissections of the disc, Canad. J.Math. 17 (1965), 302-317. 
[10] G. Chapuy, M. Marcus, G. Schaeffer, A bijection for rooted maps on orientable surfaces, SIAM J. Discrete Math. 23 (2009), 1587-1611.

[11] Z.C. Gao, The number of rooted 2-connected triangular maps on the projective plane, J. Combin. Theory Ser. B 53 (1991), 130-142.

[12] Z.C. Gao, The asymptotic number of rooted 2-connected triangular maps on a surface, J. Combin. Theory Ser. B 54 (1992), 102-112.

[13] Z.C. Gao, J.Y. Wang, Exact enumeration of rooted 3-connected triangular maps on the projective plane, Discrete Appl. Math. 141 (2004), 149-159.

[14] Z.C. Gao, The number of rooted maps with a fixed number of vertices, Ars Combinatoria 35 (1993), 151-159.

[15] Z.C. Gao, The number of degree restricted maps on a surface, Discrete Math. 123 (1993), 47-63.

[16] I.P. Goulden, D.M. Jackson, Combinatorial Enumeration, Wiley, 1983, 17-18.

[17] V.A. Liskovets, Some asymptotic estimates for planar Eulerian maps, Combin. Prob. and Comput. 5 (1996), 131-138.

[18] Yanpei Liu, Enumerative Theory of Maps, Kluwer, Boston, 1999.

[19] Yanpei Liu, Rectilinear Embeddings:Theory and Methods (in Chinese), Science Press, Beijing, 1994.

[20] Yanpei Liu, A polyhedral theory on graphs, Acta Math. Sinica 10 (1994), 136-142.

[21] Yanpei Liu, Combinatorial invariants on graphs, Acta Math. Sinica 11 (1995), 211220.

[22] A. Mednykh, R.Nedela, Enumeration of unrooted maps of a given genus, J. Combin. Theory Ser. B 96 (2006), 706-729.

[23] A. Mednykh, R. Nedela, Enumeration of unrooted hypermaps of a given genus, Discrete Math. 310 (2010), 518-526.

[24] R.C. Mullin, P.J. Schellenberg, The enumeration of c-nets via quadrangulations, J. Combin. Theory 4 (1968), 259-276.

[25] Han Ren, Yanpei Liu, On the number of regular maps on the projective plane, Utilitas Mathematica 56 (1999), 201-209.

[26] Han Ren, Yanpei Liu, The number of loopless 4-regular maps on the projective plane, J. Combin. Theory Ser. B 84 (2002), 84-99.

[27] Han Ren, Yanpei Liu, Enumeration of near-4-regular maps on the sphere and torus, Discrete Appl. Math. 110 (2001), 273-288.

[28] Han Ren, Yanpei Liu, 4-regular maps on the klein bottle, J. Combin. Theory Ser. B 82 (2001), 118-137.

[29] Han Ren, Yanpei Liu, Zhaoxiang Li, Enumeration of 2-connected 4-regular maps on the plane, Euro. J. Combin. 23 (2002), 93-111.

[30] W.T. Tutte, A theory of 3-connected graphs, Konink. Nederl. Akad. Van W. Pro. 64 (1961), 441-455. 
[31] W.T. Tutte, Graph Theory, Addson-Wesley, 1984.

[32] W.T. Tutte, A census of planar maps, Canad. J. Math. 15 (1963), 249-271.

[33] W.T. Tutte, A census of slicings, Canad. J. Math. 14 (1962), 798-722.

[34] W.T. Tutte, On the enumeration of planar maps, Bull. Amer. Math. Soc. 74 (1968), $64-74$.

[35] T.R.S. Walsh, A.B. Lehman, Counting rooted maps by genus I, II, J. Combin.Theory B 13 (1972), 122-141, 192-21.

[36] T.R.S. Walsh, A. Giorgetti, A. Mednykhc, Enumeration of unrooted orientable maps of arbitrary genus by number of edges and vertices, Discrete Math. 312 (2012), 26602671. 